\title{
Binary shape-stabilized phase change materials based on poly(ethylene glycol)/polyurethane composite with dual phase
} transition

Changzhong Chen, ${ }^{* a, b}$ Jun Chen, ${ }^{\mathrm{a}, \mathrm{b}}$ Yifan Jia, ${ }^{\mathrm{c}}$ Paul D. Topham ${ }^{\mathrm{d}}$ and Linge Wang ${ }^{* \mathrm{c}}$

${ }^{a}$ School of Chemistry, Biology and Environmental Engineering, Xiangnan University, Chenzhou 423000, China

${ }^{\mathrm{b}}$ Hunan Provincial Key Laboratory of Xiangnan Rare-Precious Metals Compounds Research and Application, Xiangnan University, Chenzhou 423043, China

${ }^{c}$ South China Advanced Institute for Soft Matter Science and Technology, South China University of Technology, Guangzhou 510640, China.

${ }^{\mathrm{d}}$ Aston Institute of Materials Research, Aston University, Birmingham, B4 7ET, UK

\begin{abstract}
Novel binary shape-stabilized composite phase change materials (CPCMs) have been successfully prepared using a crosslinked polyurethane (PU) copolymer with a solid-solid phase transition as the supporting framework for loading additional ('free') poly(ethylene glycol) (PEG). The PU copolymer was synthesized by a two-step method using 2-hydroxypropyl- $\beta$-cyclodextrin $(\mathrm{Hp}-\beta-\mathrm{CD})$ as a chain extender and PEG as a soft segment. The composition, morphology, phase transition behavior and thermal properties of the prepared CPCMs have been elucidated by a wide range of techniques. Investigation of FTIR spectra and SEM images reveal that the 'free' PEG and the PU copolymer network within the CPCMs have good compatibility and high affinity due to intermolecular interactions and capillary forces. Due to the dual phase transition of the CPCMs (the solid-liquid phase transition of 'free' PEG and solid-solid phase transition of the PU) occurring within the same,
\end{abstract}

\footnotetext{
* Corresponding authors. C. Chen, Email: czchen@126.com, Tel: +86-735-2653128;

L. Wang, Email: lingewang@ scut.edu.cn, Tel: +86-20-22236077
} 
narrow temperature window, the CPCMs have far higher heat storage density compared with that of traditional shape-stabilized PCMs with the same PEG content. Importantly, thermal cycling and thermogravimetric analysis (TGA) show that the CPCMs have good reusability and excellent thermal stability for potential use in thermoregulation or energy storage applications.

Keywords: shape-stabilized phase change material; poly(ethylene glycol); polyurethane; latent heat storage; dual phase transition

\section{Introduction}

Given the acute shortage of fossil fuels and ravenous demand of clean energy globally, latent heat storage (LHS) has recently attracted significant attention [1-3]. Phase change materials (PCMs) are extensively used in LHS systems due to their ability to store and release significant amounts of thermal energy repeatedly. PCMs have several advantages such as high heat storage density and small volume change on melting/freezing, so they are expected to play significant roles in many applications including solar energy harnessing, energy efficient buildings, central air-conditioning systems, and thermoregulating textiles [4-8].

Solid-liquid PCMs have been recognized as the most favorable candidates in LHS systems and applied extensively in various areas due to their advantages over solid-solid PCMs, such as higher heat of fusion, wider variety and lower cost $[9,10]$. Despite this, the direct utilization of solid-liquid PCMs as LHS systems is often subject to restrictions owing to their fluidity and leakage in the molten state. The common method to solve the problem is to prepare shape-stabilized (so-called 'form-stable') PCMs in which solid-liquid PCMs are loaded on a supporting framework material [11]. Owing to various noncovalent interactions (e.g., hydrogen bonds, dipole-dipole interactions, Van der Waals forces and capillary forces) between solid-liquid PCMs and the supporting material, shape-stabilized PCMs can conserve their solid form at even higher temperatures than the phase transition of solid-liquid PCMs. 
Over the last few decades, various inorganic materials and polymers (and combinations thereof) have been used as supporting materials to load solid-liquid PCMs. The most common inorganics include a variety of carbon materials (e.g., expanded graphite [12], activated carbon [10,13], carbon nanotubes [14], graphene and graphene oxide $[15,16])$, metallic and nonmetallic oxides (e.g., $\mathrm{TiO}_{2}[11]$ and $\mathrm{SiO}_{2}$ [17]) and building materials [18]. A wide range of polymers [e.g., cellulose and derivatives [19,20], poly(methyl methacrylate) (PMMA) [21], high density polyethylene (HDPE) [22], poly(D,L-lactide) (PLA) [23], polyurethane (PU) [24] and other microcapsule shells $[9,25]]$ have also been used for loading solid-liquid PCMs.

Traditional supporting materials in shape-stabilized PCMs are generally substances with high melting point and are inactive in the phase transition temperature range of the loaded solid-liquid PCM. Crucially, they not only make zero contribution to the latent heat storage of shape-stabilized PCMs, but also have a negative effect on the crystallization of the solid-liquid PCM. As a consequence, the heat storage density of typical shape-stabilized PCMs is considerably lower than the corresponding pristine solid-liquid PCM. In order to solve the problem, we reported novel shape-stabilized composite PCMs using synthesized polymeric glucose/diphenylmethane diisocyanate/PEG solid-solid PCMs to load poly(ethylene glycol) (PEG) [26]. Due to the synergetic phase transition of the supporting material and the loaded PEG, the obtained shape-stabilized PCMs exhibited a far higher enthalpy than traditional shape-stabilized PCMs. Similarly, shape-stabilized PCMs with high phase change enthalpy based on hexadecanol/phase change PU [27], paraffin/cross-linking SSPCM [28], PEG/thermosetting SSPCM [29] composites were successively prepared. Regrettably, all the above work only focused on the thermal properties of the composite PCMs, but further study of the surface morphology of the composite PCMs and synergetic phase transition process of the solid-solid PCMs/solid-liquid PCMs in the composite PCMs are also important and necessary because these factors can influence the shape-stabilization and heat storage properties of composite PCMs.

In this study, polymers have been designed for use as thermoregulation or energy storage materials. Accordingly, a new crosslinked PU copolymer with solid-solid 
phase transition has been synthesized using 2-hydroxypropyl- $\beta$-cyclodextrin

$(\mathrm{Hp}-\beta-\mathrm{CD})$ as a chain extender and PEG as a soft segment, and then binary shape-stabilized composite PCMs (CPCMs) were prepared using the synthesized PU copolymer as a matrix to load various amounts of PEG. Since the CPCMs can undergo dual phase transition from PEG (solid-liquid phase transition) and PU copolymer (solid-solid phase transition) in a similar phase change temperature range, they are expected to have high heat storage density compared with traditional shape-stabilized PCMs with the same PCM content. In addition to a detailed study of the composition, crystallization and thermal storage capability of the CPCMs, their surface morphology [by scanning electron microscopy (SEM)] and the phase transition behavior [observed by in situ polarized light optical microscopy (POM)] are described in this research.

\section{Experimental}

\subsection{Materials}

2-Hydroxypropyl- $\beta$-cyclodextrin (Hp- $\beta$-CD), diphenylmethane diisocyanate (MDI) and anhydrous $\mathrm{N}, \mathrm{N}$-dimethylformamide (DMF, AR) were purchased from Aladdin Chemistry Co., Ltd., China. Ethyl cellulose (EC) was produced by Tianjin Guangfu Fine Chemical Research institute, China. PEG $\left(M_{\mathrm{n}} \sim 8000 \mathrm{Da}\right)$ was produced by Amresco Co., USA. Hp- $\beta$-CD, EC and PEG powders were dried in a vacuum oven at $100{ }^{\circ} \mathrm{C}$ for $6 \mathrm{~h}$. MDI was heated in a vacuum oven at $60^{\circ} \mathrm{C}$ for $2 \mathrm{~h}$ and then filtered by a heated filter. DMF and ethanol was used without further treatment.

\subsection{Preparation of shape-stabilized CPCMs}

PU copolymer with crosslinked networks was synthesized firstly similar to previous studies [30,31]. MDI solution (6 mmol in DMF) was dropped slowly into a stirring PEG solution (3 mmol in DMF) in a three necked round bottom flask, which was then lowered into a thermostatic oil-bath at $90{ }^{\circ} \mathrm{C}$ under nitrogen atmosphere for $6 \mathrm{~h}$. Then, a Hp- $\beta$-CD solution (0.857 mmol in DMF) was prepared and dropped into the reaction system with stirring. After $24 \mathrm{~h}$, the mixture was poured into an unsealed beaker for 3 days of thermal curing at $80{ }^{\circ} \mathrm{C}$ in an oven. Finally, the yellowish solid products were obtained after washing with ethanol three times and drying at $80{ }^{\circ} \mathrm{C}$ in 
the oven. Solubility tests of the synthesized material were performed at $20{ }^{\circ} \mathrm{C}$ in various solvents, demonstrating that the solubility of the crosslinked copolymer in all common solvents trialed was lower than $0.01 \mathrm{~g} / 100 \mathrm{ml}$.

The shape-stabilized CPCMs in this work were prepared as follows. PEG solution was obtained by dissolving $1 \mathrm{~g}$ PEG in $10 \mathrm{ml}$ ethanol at $80{ }^{\circ} \mathrm{C}$ (with a backflow device to prevent evaporation). Different amounts of PU copolymer were added to the PEG solution with vigorous stirring for $2 \mathrm{~h}$. The mixture was put into an unsealed beaker at $80{ }^{\circ} \mathrm{C}$ to evaporate the solvent. A series of CPCMs with $30 \mathrm{wt} \%-80 \mathrm{wt} \%$ PEG (with respect to the total weight) were fabricated. The synthetic route of the CPCMs is depicted in Scheme 1.

Leakage tests were performed to verify the shape-stabilized properties of the CPCMs. The as-prepared CPCMs and pristine PEG (as a reference) were simultaneously put in the oven at $80{ }^{\circ} \mathrm{C}$. The samples remained in the oven for five additional minutes after the pure PEG powders completely melted. Then, all the samples were taken out from the oven to cool to room temperature. These heating-cooling cycles were carried out 10 times. CPCMs that did not produce melted PEG during testing were recognized as shape-stabilized CPCMs. The results showed that the maximum PEG loading into the PU copolymer without leakage of melt PEG was $60 \mathrm{wt} \%$. The shape-stabilized CPCMs with $30 \mathrm{wt} \%, 40 \mathrm{wt} \%, 50 \mathrm{wt} \%$ and $60 \mathrm{wt} \%$ PEG are labeled as CPCM-30, CPCM-40, CPCM-50 and CPCM-60, respectively. For comparison, ethyl cellulose/PEG (EC/PEG) composite PCMs with 30 wt\% - 60 wt\% PEG (EC/PEG-30, EC/PEG-40, EC/PEG-50, and EC/PEG-60, respectively) were also prepared by solution casting, as described above.

\subsection{Characterization}

Fourier transformed infrared (FT-IR) spectroscopy was performed using a Nicolet 760 spectrometer (Nicolet Co., USA) between $4000 \mathrm{~cm}^{-1}$ and $400 \mathrm{~cm}^{-1}$. Homogeneous mixtures of each sample with $\mathrm{KBr}$ powder were pressed into small pellets before testing. 
The morphology of samples was observed using a Phenom Pro G3 scanning electron microscopy (SEM, Phenom, Holland) operating at $10 \mathrm{kV}$. Samples were thinly sputter-coated with gold before SEM observation.

$\mathrm{X}$-ray diffraction (XRD) analyses were performed using a wide-angle X-ray diffractometer (D8 Advance, Bruker-AXS, Germany) with a $\mathrm{Cu} \mathrm{K} \alpha$ radiation source. All diffraction data were acquired in the range of $5^{\circ}<2 \theta<50^{\circ}$ with a collection time of $0.1 \mathrm{~s}$ per step.

POM images of PEG, PU and CPCMs at room temperature and at phase transition process were obtained by a polarized light optical microscope (XPN-300E, Shanghai Changfang Optical Instrument Co., Ltd, China) equipped with a video-camera and a hot stage. The POM images of samples at melting process were obtained from room temperature to $80{ }^{\circ} \mathrm{C}$ at a heating rate of $5{ }^{\circ} \mathrm{C} / \mathrm{min}$. And the POM images of samples at crystallization process were obtained via two steps. Firstly, the sample was heated to $80{ }^{\circ} \mathrm{C}$ quickly with a heating rate of $12{ }^{\circ} \mathrm{C} / \mathrm{min}$ and then kept at $80{ }^{\circ} \mathrm{C}$ for $5 \mathrm{~min}$. Secondly, the sample was naturally cooled from $80{ }^{\circ} \mathrm{C}$ to $40^{\circ} \mathrm{C}$ and the POM images of samples were recorded at $40{ }^{\circ} \mathrm{C}$ at different times.

Simultaneous Thermal Analysis Apparatus (STA 449 F3 Jupiter®, Netzsch, Germany) was used to record the differential scanning calorimetry (DSC) thermograms and thermogravimetric analysis (TGA) profiles under $\mathrm{N}_{2}$ atmosphere. A small amount of sample (ca. $10 \mathrm{mg}$ ) was placed in an aluminum pan, sealed, and four heat-cool cycles were performed between $20{ }^{\circ} \mathrm{C}$ and $100{ }^{\circ} \mathrm{C}$ at a scanning rate $2{ }^{\circ} \mathrm{C} / \mathrm{min}$. All DSC thermograms were recorded from the second thermal cycle. Thermal property data (phase change enthalpy and phase change temperature) were reported as average values of the recorded DSC curves with standard deviations. TGA measurements were performed in the range of $25-600{ }^{\circ} \mathrm{C}$, under nitrogen flow, at a heating rate of $10{ }^{\circ} \mathrm{C} / \mathrm{min}$.

Thermal cycling tests of the shape-stabilized CPCMs were performed on a hot stage. Samples were loaded into aluminum pans and placed on the hot stage with a program of 100, 500 and 1000 heating-cooling thermal cycles in the range of 25$100{ }^{\circ} \mathrm{C}$. The time of each heating-cooling thermal cycle was set at $20 \mathrm{~min}$. DSC 
thermograms of the cycled CPCMs were also performed in the range of $20-100{ }^{\circ} \mathrm{C}$ at a $2{ }^{\circ} \mathrm{C} / \mathrm{min}$ scanning rate.

\section{Results and discussion}

From our previous studies [31,32], crosslink density was shown to be a vital factor influencing the phase transition enthalpy of crosslinked polymeric solid-solid PCMs. In this work, a series of PU copolymers with different crosslink density were synthesized by varying the reactant molar ratios. It was found that the PU copolymer with molar ratio of 7:14:2 (PEG : MDI : Hp- $\beta-\mathrm{CD}$ ) had the highest phase change enthalpy and was therefore selected as the supporting material of the CPCMs.

\subsection{FTIR study}

The molecular structure of the reactants, PU copolymer and the compositions of CPCMs were characterized by FTIR as shown in Figure 1. The characteristic bands of $\mathrm{Hp}-\beta-\mathrm{CD}$ at 3412,1155 , and $1028 \mathrm{~cm}^{-1}$ correspond to $\mathrm{O}-\mathrm{H}$ stretching, $\mathrm{C}-\mathrm{O}$ bending/stretching, and $\mathrm{C}-\mathrm{O}$ bending vibrations, respectively [33]. From the FTIR spectra of pristine PEG, salient characteristic bands at 3432, 1281 and $1242 \mathrm{~cm}^{-1}$ are assigned to $\mathrm{O}-\mathrm{H}$ stretching and bending vibrations. The characteristic absorption bands at 2889, 1467, 963 and $842 \mathrm{~cm}^{-1}$ are attributed to the stretching/bending of $\mathrm{C}-\mathrm{H}$ bonds [33]. The bands at 1151, 1110 and $1060 \mathrm{~cm}^{-1}$ (with a maximum at $1110 \mathrm{~cm}^{-1}$ ) were observed due to the $\mathrm{C}-\mathrm{O}-\mathrm{C}$ stretching vibration [13]. The prominent band of MDI observed at $2276 \mathrm{~cm}^{-1}$ corresponds to the stretching vibration of the NCO group. In the case of PU copolymer, most of the characteristic bands of Hp- $\beta-\mathrm{CD}$ and PEG with minor shifts were observed. The absorption band of the NCO group vanished and the band at $3300-3500 \mathrm{~cm}^{-1}$ corresponding to $\mathrm{O}-\mathrm{H}$ stretching become weaker compared with pristine Hp- $\beta-\mathrm{CD}$ and PEG, as expected. Moreover, new characteristic bands at 1727,1540 and $1597 \mathrm{~cm}^{-1}$ were observed, corresponding to the stretching vibration of $\mathrm{C}=\mathrm{O}, \mathrm{N}-\mathrm{H}$ and benzene rings, respectively. These results show that crosslinked copolymer was successfully obtained by the covalent bonding of PEG, MDI and Hp- $\beta-C D$. 
The solubility tests indicate that the synthesized PU copolymer was insoluble in common polar solvents (deionized water, DMF, tetrahydrofuran, dimethylsulfoxide, methyl alcohol, ethyl alcohol, acetone, etc.) and non-polar solvents (petroleum ether, dichloromethane, chloroform, tetrachloromethane, benzene, toluene, etc.), also suggesting the formation of a chemically-crosslinked network in the PU copolymer.

In the FTIR spectra of the CPCMs, most characteristic bands from PEG also appear with only a slight shift. For instance, the bands of $\mathrm{C}-\mathrm{O}-\mathrm{C}$ and $-\mathrm{CH}_{2}$ stretching shift from $1151 \mathrm{~cm}^{-1}$ and $2889 \mathrm{~cm}^{-1}$ to $1149 \mathrm{~cm}^{-1}$ and $2885 \mathrm{~cm}^{-1}$, respectively. The characteristic bands at 1727,1540 and $1597 \mathrm{~cm}^{-1}$ in the PU copolymer also exist in the CPCM FTIR spectra with a reduction in intensity. The red-shift in wavenumber of the functional groups for CPCMs suggests that there are some noncovalent bond interactions such as intermolecular hydrogen bonds and dipole-dipole interactions between PEG and PU copolymer. These interactions between PEG and PU copolymer have positive impact on preventing the leakage of the melted PEG from the supporting materials.

\subsection{Morphology of the shape-stabilized CPCMs}

The morphologies of pristine PEG and the PU copolymer observed by SEM are represented in Figure 2. PEG powders display a relatively smooth surface with few pores (tens of microns in diameter) from the SEM images with low and high resolution. Interestingly, the synthesized PU copolymer possesses a lamellar or fibrillar structure (the width of the fibrils and lamellar spaces being several microns). The inherent spacing within the lamellar stacks of the PU copolymer are expected to facilitate high encapsulation of PEG and maintain the molten PEG within the voids during the fusion process.

Figure 3 shows representative SEM images of the CPCMs with varied mass ratio. When the PEG content is low $(\leq 40 \mathrm{wt} \%$, Fig. 3a,b), some pores remain on the surface of the CPCMs, indicating that the lamellar voids of the PU copolymer have not been completely filled by PEG. The number of pores and their size clearly decrease with increase in PEG content (Fig. 3c). In the case of CPCM-60 (Fig. 3d), the surface morphology becomes relatively smooth as the lamellar spaces of the PU copolymer 
become more efficiently filled by PEG. Crucially, PEG dispersed well among the lamellar spaces of the PU copolymer and no visual phase separation occurred on the surface of any of the CPCMs. This demonstrates that PEG has good compatibility and high affinity with our PEG-containing PU polymer. From the FTIR spectra and SEM images, it is inferred that the shape-stabilization of molten PEG in the CPCMs is attributed to the combination of a physical supporting effect of lamellar/fibrillar PU copolymer and noncovalent electronic interactions, such as hydrogen bonds and dipole-dipole interactions between PEG and the PU copolymer.

\subsection{Crystalline properties and phase transition behaviors of the shape-stabilized} CPCMs

Figure 4 shows the XRD traces of Hp- $\beta$-CD, PEG, PU copolymer and CPCM series. Neat Hp- $\beta-C D$ has no diffraction peaks, demonstrating its amorphous nature. PEG, a linear aliphatic polyether with highly symmetrical and non-branched structure, has good crystalline properties due to the efficient packing of the molecular chains, and exhibits sharp characteristic diffraction peaks at $2 \theta=19.3^{\circ}$ and $24.6^{\circ}$. Two main sharp diffraction peaks at the same positions as those of PEG also exist and no additional new peaks appear in the XRD of PU copolymer, suggesting that the crystallinity in the PU copolymer networks arises from the PEG segments. Compared with pristine PEG, the diffraction peaks of PU copolymer have lower intensity, contributing to the decline of content and the restriction of free movement of PEG segments in the PU network [30,31,34,35].

In the case of CPCMs, they also exhibit two main characteristic peaks at the same positions, and other weak diffraction peaks of pristine PEG become much weaker in the CPCMs. The intensity of all diffraction peaks has a few increases with the increase of 'free' PEG content of CPCMs, but it is lower than that of pristine PEG. Obviously, the dispersion of PEG in the crosslinked network of PU copolymer also goes against the crystallinity of CPCMs to some extent.

POM was employed to further probe the crystalline morphology of the samples further. Representative POM images of PEG, PU copolymer and the CPCM series at room temperature are given in Figure 5. Neat PEG exhibits typical spherulitic 
morphology with spherulites reaching millimeters in size, and the cross-extinction patterns with black color can be clearly observed under the cross-polarized light (Fig 5a). In the case of the PU copolymer (Fig. 5b), spherulites with smaller size are observed, consistent with the lower diffraction peak intensity in the XRD data.

All of the CPCMs exhibit spherulites under polarized light (Fig. 5c-f) where the spherulite size changes with PEG content. More specifically, larger spherulites from PEG and smaller spherulites from the PU copolymer coexist when the PEG content is relatively low (Fig. 5c,d). When the PEG content increases, the spherulites become more uniform and are increasingly similar to those of pristine PEG (Fig. 5e,f). From the SEM image in Fig. 3c,d, 'free' PEG almost filled in the space of the CPCM's network when PEG content is high, thus PEG plays a dominant role in the crystallization process of CPCMs at this point.

In order to intuitively investigate the phase transition behaviors of the CPCMs, in-situ polarized light optical microscopy was used to observation the POM images of samples during the melting/crystallization process. Figure 6 depicts in-situ POM images of neat PEG, PU copolymer and CPCM-40 during heating process. The spherulite melting range (indicated by the obvious change in brightness to complete disappearance of the spherulites) for samples measured from POM are listed in Table 1. POM images of neat PEG have no obvious changes below $58{ }^{\circ} \mathrm{C}$. The color of the POM image diminished and the brightness of POM image dimmed at $58^{\circ} \mathrm{C}$. A dark image appeared quickly when the temperature reached $60{ }^{\circ} \mathrm{C}$, whilst the PEG in the glass slide concomitantly transformed from a white solid film to transparent liquid drops. The narrow melting range of neat PEG suggests that the spherulites grew by a single crystallization mechanism.

Unlike pristine PEG, no liquid phase was observed for PU copolymer or CPCM-40, during the entire heating process (from room temperature to $80^{\circ} \mathrm{C}$ ) suggesting a true solid-solid phase transition in the PU copolymer and good shape-stabilization performance of CPCM-40. As shown in Figure 6, the POM images of PU copolymer exhibit regular spherulitic patterns with no obvious changes occurring below $55{ }^{\circ} \mathrm{C}$. The color of the PU copolymer spherulites faded gradually at $55{ }^{\circ} \mathrm{C}$, indicating the 
phase transition of the PU copolymer as the crystals melt to the amorphous state. When the temperature reached $60{ }^{\circ} \mathrm{C}$, the spherulites of the PU copolymer completely vanished. The melting range of the PU copolymer was broader than that of pristine PEG from Table 1 (which is also confirmed by the following DSC thermograms measurement), indicating the crosslinked network has an impact on the crystallization mechanism of the somewhat constrained PEG segments.

In the case of CPCM-40, the spherulites faded gradually at $57^{\circ} \mathrm{C}$, and the color fading became more prevalent at $60{ }^{\circ} \mathrm{C}$. The spherulites of CPCM-40 completely disappeared at $62{ }^{\circ} \mathrm{C}$. The melting range of CPCM-40 is also wider than that of PEG, again suggesting that the supporting framework influences the crystallization mechanism of the 'free' PEG in the CPCMs.

In order to deeper explore the probable mechanism of crystallization, the crystallization behavior of neat PEG, PU copolymer and CPCM-40 was investigated. Figure 7 gives the POM images of spherulite growth for PEG, PU copolymer and CPCM-40. During the entire crystallization process, all the POM images taken for pristine PEG had only one spherulite with clear cross-extinction patterns in the field of view. The PEG spherulite grew symmetrically from the centre to the edge quickly (the growth speed of spherulite was about $10 \mu \mathrm{m} / \mathrm{s}$ ). There are two stages during the kinetic process of spherulite formation: nucleation and growth. Furthermore, the nucleation step of a single spherulite can proceed via one of two mechanisms: homogeneous or heterogeneous nucleation. It is evident that the crystallization of pristine PEG should proceed via homogeneous nucleation. The initial aggregate (namely crystal nucleus) forms from a small number of aligned, folded PEG chains, before growing at a constant rate until impingement (with other vicinal growing spherulites) occurs. The growth process of crystal nucleus cannot be observed due to the lower magnification times of the polarizing optical microscope, but the process of growth of the spherulite can be observed clearly by POM. As shown in Fig. 7, the pristine PEG spherulite grows quickly once its crystal nucleus formed. It is worth noting that the diameter of PEG spherulite is beyond $500 \mu \mathrm{m}$ in the last POM image 
of PEG, which is significantly larger than commonly observed polymer spherulites, which have a typical size of $0.5-100 \mu \mathrm{m}$.

Compared with pristine PEG, the number of spherulites for the PU copolymer increased significantly, and its growth rate was much slower (the growth speed of spherulite was only about $0.5 \mu \mathrm{m} / \mathrm{s}$ ). It is concluded that the constraints exerted by the PU copolymer not only influenced the nucleation process of PEG chains, but also influenced their growth. The hard segments restricted the movement of PEG chains, which results in the decrease of growth rate. Moreover, the spherulite size of PU copolymer was smaller than that of pristine PEG, revealing PEG segments in PU copolymer have more unperfected crystal structure than that of PEG. Most of spherulites of PU copolymer have the same size at the same crystallization time, suggesting the homogeneous nucleation of PU copolymer.

The growth rate of spherulites of CPCM-40 also decreased and the number of spherulites increased compared with pristine PEG. Unlike both pristine PEG and the PU copolymer, the spherulites of CPCM-40 have a broad distribution of sizes at a given crystallization time. This suggests that there is a different crystallization mechanism occurring in the PEG/PU blend than in the pristine polymer systems, with a combination of homogeneous and heterogeneous crystallization.

\subsection{Phase change heat storage properties of the shape-stabilized CPCMs}

Differential scanning calorimetry (DSC) provides valuable information about the phase change behavior of materials. The second heating and cooling thermograms of PEG, PU copolymer and CPCMs are shown in Figure 8. The relevant phase transition enthalpies of samples were obtained by integration of the DSC peaks above the base line, and their phase transition temperatures correspond to the onset temperatures (rather than peak temperatures). The corresponding thermal property data (melting temperature $T_{m}$, melting enthalpy $\Delta H_{m}$, crystallization temperature $T_{c}$, and crystallization enthalpy $\Delta H_{c}$ ) are shown in Figure 9. The salient melting peak with $T_{m}$ of $60.4{ }^{\circ} \mathrm{C}$ and $\Delta H_{m}$ of $148.12 \mathrm{~J} / \mathrm{g}$ and crystallizing peak with $T_{c}$ of $47.6^{\circ} \mathrm{C}$ and $\Delta H_{c}$ of $139.30 \mathrm{~J} / \mathrm{g}$ appear in the heating and cooling DSC curve of PEG, attributed to its phase transition between solid and molten PEG. 
The PU copolymer also exhibits a sharp endothermic peak and exothermic peak near that of pristine PEG, and its $T_{m}, \Delta H_{m}, T_{c}$, and $\Delta H_{c}$ are $57.5^{\circ} \mathrm{C}, 96.20 \mathrm{~J} / \mathrm{g}, 44.2^{\circ} \mathrm{C}$, and $91.1 \mathrm{~J} / \mathrm{g}$, respectively. The decrease in phase change temperature and enthalpy is caused by the lower crystallinity in the PU copolymer in line with XRD data and POM images.

It is clear that there are two types of phase transition occurring in the CPCMs during the heating/cooling processes. As shown in Figure 10, the PEG chains/segments in the CPCMs undergo transformation between an ordered arrangement and disordered entangled 'melt' during heating/cooling, corresponding to the phase transition of PEG (between solid crystalline phase and liquid phase) and PU copolymer (between crystalline phase and amorphous state).

However, from Fig. 8, the heating and cooling thermograms of all CPCMs exhibit single endothermic and exothermic peak in a similar temperature range to PEG even at a heating/cooling rate of only $2{ }^{\circ} \mathrm{C} / \mathrm{min}$. In other words, the CPCMs comprising PU copolymer and 'free' PEG boast dual phase transition in a single, relatively narrow, temperature range, which is in accordance with those in Ref. [26,29]. Though polymeric solid-solid PCMs containing PEG segments are used as the supporting framework in all the reported shape-stabilized PCMs based on solid-liquid PCM/solid-solid PCM composite [26-29], there are two types of loaded solid-liquid PCMs: PEG [26,29] or non-PEG solid-liquid PCMs [27,28]. It is found that there are only single endothermic and exothermic peak in the DSC thermograms of PEG/solid-solid PCM composites and multi peaks in the DSC thermograms of non-PEG solid-liquid PCMs/solid-solid PCM composites, which is determined by the phase transition temperature range of loaded solid-liquid PCMs and solid-liquid PCMs in the CPCMs. When the two components (namely PEG and the solid-solid PCMs) undergo phase transitions in the similar temperature range, the DSC thermograms of them would be completely overlapped and exhibit single endothermic and exothermic peak. In contrast, when the two components (namely non-PEG solid-liquid PCMs and the solid-solid PCMs) undergo phase transitions in the different temperature range, the DSC thermograms of them would exhibit multi 
endothermic and exothermic peaks. From the perspective of thermal properties, the shape-stabilized PCMs based on solid-liquid PCM/solid-solid PCM composite with single endothermic/exothermic peak have better synergetic and cumulative effect by the two components.

Figure 9 shows that $\Delta H_{m}$ and $\Delta H_{c}$ of all the CPCMs falls in between the values of PEG and PU copolymer (in the range of $103-124 \mathrm{~J} / \mathrm{g}$ ). For comparison, the phase change properties of EC/PEG composite PCMs (from DSC analysis) are also included in Figure 9. Clearly, $\Delta H_{m}$ and $\Delta H_{c}$ of the prepared CPCMs in this work are far higher than those of EC/PEG PCMs with the same PEG content, which indicates that the PU copolymer supporting material can significantly enhance the latent heat storage density of the shape-stabilized PCMs.

Theoretically, the phase change enthalpies of traditional shape-stabilized PCMs containing PEG are calculated by Equations 1 and 2:

$$
\begin{aligned}
& \Delta H_{m-P C M}^{T}=\Delta H_{m-P E G} \times \omega_{P E G} \\
& \Delta H_{c-P C M}^{T}=\Delta H_{c-P E G} \times \omega_{P E G}
\end{aligned}
$$

where ${ }^{\Delta H_{m-P C M}^{T}}$ and $\triangle H_{c-P C M}^{T}$ are the theoretical melting and crystallizing enthalpies of the traditional shape-stabilized PCM, respectively; ${ }^{\Delta H_{m-P E G}}$ and ${ }^{\Delta H_{c-P E G}}$ are the melting and crystallizing enthalpies of PEG; and $\omega_{P E G}$ is the mass fraction of PEG in the shape-stabilized PCMs.

In contrast, $\Delta H_{m}$ and $\Delta H_{c}$ of the CPCMs in this study came from the simultaneous dual phase transition of PEG and PU copolymer. Thus, the theoretical phase change enthalpies of the CPCMs are the sum of those of PEG and PU copolymer in the CPCMs, which can be calculated by Equations 3 and 4:

$$
\begin{aligned}
& \Delta H_{m-C P C M}^{T}=\Delta H_{m-P E G} \times \omega_{P E G}+\Delta H_{m-P U} \times \omega_{P U} \\
& \Delta H_{m-C P C M}^{T}=\Delta H_{c-P E G} \times \omega_{P E G}+\Delta H_{c-P U} \times \omega_{P U}
\end{aligned}
$$

where $\Delta H_{m-C P C M}^{T}$ and $\Delta H_{c-C P C M}^{T}$ are the theoretical melting and crystallizing enthalpies of CPCM, respectively; $\Delta H_{m-P U}$ and $\Delta H_{c-P U}$ are the theoretical melting and crystallizing 
enthalpies of PU copolymer, respectively; $\omega_{P U}$ is the mass fraction of PU copolymer in the CPCM, where $\omega_{P E G}+\omega_{P U}=1$.

By comparison, the theoretical values of CPCMs are higher than those of traditional shape-stabilized PCMs from Equations 1 to 4 because $\Delta H_{m-P U}$ and $\Delta H_{c-P U}$ are nonzero parameters. Compared with traditional supporting material, the PU copolymer with a solid-solid phase transition used in this work is a better candidate for enhancing the heat storage density of shape-stabilized PCMs.

Enthalpy efficiency (E) of shape-stabilized PCMs can be used to evaluate the disparity between experimental and theoretical enthalpy values. Simply, E is the ratio between $\Delta H_{m}\left(\Delta H_{c}\right)$ of the CPCMs and the corresponding theoretical values. Here, two kinds of CPCM enthalpy efficiency can be obtained (as shown in Figure 11) corresponding to the two kinds of theoretical enthalpy values. It is found that $\Delta H_{m}$ and $\triangle H_{c}$ of the CPCMs are in the narrow range of $97.4 \%-99.7 \%$ of $\triangle H_{m-C P C M}^{T}$ and $\Delta H_{c-C P C M}^{T}$, suggesting only a slight deviation of the experimental values from the theoretical values.

In contrast, $\Delta H_{m}$ and $\Delta H_{c}$ of the CPCMs are far higher than $\Delta H_{m-P C M}^{T}$ and $\Delta H_{c-P C M}^{T}$ of traditional shape-stabilized PCMs with the same PEG loading. More specifically, $\Delta H_{m}$ and $\Delta H_{c}$ of the CPCMs are in the range of $140 \%-250 \%$ of $\Delta H_{m-P C M}^{T}$ and $\Delta H_{c-P C M}^{T}$. There is a clear trend in enthalpy efficiency of the CPCMs, decreasing with increasing PEG loading. The phase change enthalpies of CPCMs have a greater enhancement when the 'free' PEG content in the CPCMs is lower. The theoretical enthalpy values $\left(\Delta H_{m-P C M}^{T}\right.$ and $\left.{ }^{\Delta H_{c-P C M}^{T}}\right)$ of traditional shape-stabilized PCMs are generally higher than their corresponding experimental enthalpy values with the same PCMs loading [5-8,10]. As a consequence, it is not a particular case that the CPCMs have preferable heat storage density than EC/PEG composite PCMs.

3.5 Reusability and thermal stability of the shape-stabilized CPCMs 
Considering the economic and environmental benefits, the reusability of PCMs is one of most important factors in determining their fate in widespread use. Accelerated thermal cycling tests can be used to explore the variations of phase change performance of the prepared CPCMs, giving an indication of their expected lifetime. Figure 12 shows an SEM image and FTIR spectrum of CPCM-60 after 1000 thermal cycles. From Fig. 12a, no visible phase separation occurred on the surface of CPCM-60, revealing that molten PEG did not leak from the supporting framework during the vast number repeated cycles. In short, the morphology of CPCM-60 has no obvious changes compared with the original one shown in Fig. 3d, which demonstrates the excellent shape-stabilized performance of CPCM-60 after repeated use. Likewise, there are negligible differences between the FTIR spectra of original and thermally cycled CPCM-60 (Fig. 12b), indicating that no chemical reaction or thermal degradation occurred during the cycling process.

Figure 13 shows the DSC thermograms and thermal property data of CPCM-60 after various thermal cycles. It is found that the DSC thermogram of thermally cycled CPCM-60 almost completely overlap with the original thermogram (given in Fig. 13a), and the changes in phase change enthalpy and temperature are negligible (Fig. 13b). These results show that the prepared CPCMs could be utilized repeatedly without the decline of phase change properties in the phase change temperature interval.

The thermal stability of PCMs is also a significant indicator for their practical applications, which is often characterized by TGA. Figure 14 shows the TGA and DTG curves of Hp- $\beta$-CD, PEG, PU copolymer and the CPCMs. The TGA trace of Hp- $\beta$-CD has two degradation steps: one below $100{ }^{\circ} \mathrm{C}$ and one around $300{ }^{\circ} \mathrm{C}$. The first step with about $14 \%$ mass loss is caused by the evaporation of bound water in the cavities of $\mathrm{Hp}-\beta-\mathrm{CD}$, and the second one represents the breaking of chemical bonds in Hp- $\beta$-CD. PEG has good thermal stability with one degradation step at 376.2 $-412.8^{\circ} \mathrm{C}$; peak temperature from the DTG curve at $396.7^{\circ} \mathrm{C}$. Two successive degradation steps with different degradation rate exist in the TGA curve of the PU copolymer. The first degradation step is attributed to the breakage of hard segments 
from $\mathrm{Hp}-\beta-\mathrm{CD}$, and the second one in the temperature range of $381.7-421.0^{\circ} \mathrm{C}$ corresponds to the breakage of soft segments from PEG.

For the CPCMs, the first degradation step from PU copolymer became weaker with increasing PEG loading. The thermal degradation onset temperature of the second step from PEG was above $380{ }^{\circ} \mathrm{C}$ and the corresponding peak temperature (shown in the DTG curves) was above $400{ }^{\circ} \mathrm{C}$, higher than that of pristine PEG. The mass loss in the CPCMs at $300{ }^{\circ} \mathrm{C}$ was only $2.60 \%, 2.46 \%, 1.57 \%$ and $1.05 \%$ for CPCM-30, CPCM-40, CPCM-50 and CPCM-60, respectively. According to the results from TGA and DTG curves, the CPCMs have good thermal stability for thermal energy storage.

\section{Conclusions}

A crosslinked polyurethane copolymer comprising Hp- $\beta-C D$ and PEG linkages with a solid-solid phase transition was synthesized and used as a supporting material for loading additional 'free' PEG (which exhibits a solid-liquid phase transition). A series of shape-stabilized composite phase change materials (CPCMs) with large heat storage density, good reusability and excellent thermal stability were facilely prepared by solution casting, and the maximum PEG loading percentage in the CPCMs without leakage of molten PEG was shown to be $60 \mathrm{wt} \%$. Due to intermolecular interactions and capillary forces between free PEG and the PU copolymer, the two components have good compatibility in the CPCMs. Since the solid-liquid phase transition of PEG and solid-solid phase transition of PU copolymer occurred simultaneously in the CPCMs, the CPCMs have far higher heat storage density than that of traditional shape-stabilized PCMs with the same PEG loading percent. The strategy of preparing the novel CPCMs in this work could overcome the common problem of enthalpy decline of shape-stabilized PCMs for future use in thermoregulation or energy storage. In addition, this work further deepens understanding towards synergetic phase transition behavior of binary shape-stabilized PCMs based on solid-liquid PCM/solid-solid PCM composites.

\section{Acknowledgements}


The authors thank the financial support from National Natural Science Foundation of China (No. 21404061 and 21506065), the National Key R\&D Program of China (No.2017YFC11050003), Guangdong Innovative and Entrepreneurial Research Team Program (No. 2016ZT06C322), the key Project of Hunan Provincial Department of Education (17A199), Guangdong Natural Science Foundation (2015A030310472) and Scientific Research Foundation of Xiangnan University for High-Level Talents. PDT also thanks the State Administration for Foreign Experts Affairs and the Royal Society of Chemistry for a Visiting Researcher Program grant to China.

\section{Conflict of interest statement}

The authors declare that no conflict of interest exist.

\section{References}

[1] A. Abhat, Low temperature latent heat thermal energy storage: heat storage materials, Sol. Energy 30 (1983) 313-332.

[2] A.M. Khudhair, M.M. Farid, A review on energy conservation in building applications with thermal storage by latent heat using phase change materials, Energy Convers. Manag. 45 (2004) 263-275.

[3] P. Zhang, X. Xiao, Z.W. Ma, A review of the composite phase change materials: Fabrication, characterization, mathematical modeling and application to performance enhancement, Appl. Energy 165 (2016) 472-510.

[4] M. Kenisarin, K. Mahkamov, Solar energy storage using phase change materials, Renew. Sust. Energy Rev. 11 (2007) 1913-1965.

[5] K. Pielichowska, K. Pielichowski, Phase change materials for thermal energy storage, Progress in Materials Science 65 (2014) 67-123.

[6] D. Zhou, C.Y. Zhao, Y. Tian, Review on thermal energy storage with phase change materials (PCMs) in building applications, Appl. Energy 92 (2012) 593-605.

[7] E. Oró, A. de Gracia, A. Castell, M.M. Farid, L.F. Cabeza, Review on phase change materials (PCMs) for cold thermal energy storage applications, Appl. Energy 99 (2012) 513-533. 
[8] R. Jacob, F. Bruno, Review on shell materials used in the encapsulation of phase change materials for high temperature thermal energy storage, Renew. Sust. Energy Rev. 48 (2015) 79-87.

[9] M. Mehrali, S.T. Latibari, M.A. Rosen, A.R. Akhiani, M.S. Naghavi, E. Sadeghinezhad, H.S.C. Metselaar, M.M. Nejad, M. Mehrali, From rice husk to high performance shape stabilized phase change materials for thermal energy storage, RSC Adv. 6 (2016) 45595-45604.

[10] S.T. Latibari, M.Mehrali, M.Mehrali, A.B.M. Afifi, T.M.I. Mahlia, A.R. Akhiani, H.S.C. Metselaar, Facile synthesis and thermal performances of stearic acid/titania core/shell nanocapsules by sol-gel method, Energy 85 (2015) 635-644.

[11] Y. Wu, C. Chen, Y. Jia, J.Wu, Y. Huang, L. Wang, Review on electrospun ultrafine phase change fibers (PCFs) for thermal energy storage, Appl. Energy 210 (2018) 167-181.

[12] A. Sarı, A. Karaipekli, Preparation, thermal properties and thermal reliability of palmitic acid/expanded graphite composite as form-stable PCM for thermal energy storage, Sol. Energy Mater. Sol. Cells 93 (2009) 571-576.

[13] C. Wang, L. Feng, W. Li, J. Zheng, W. Tian, X. Li, Shape-stabilized phase change materials based on polyethylene glycol/porous carbon composite: The influence of the pore structure of the carbon materials, Sol. Energy Mater. Sol. Cells 105 (2012) $21-26$.

[14] I. Kholmanov, J. Kim, E. Ou, R.S. Ruoff, L. Shi, Continuous carbon nanotube-ultrathin graphite hybrid foams for increased thermal conductivity and suppressed subcooling in composite phase change materials, ACS Nano 9 (2015) 11699-11707.

[15] Y. Xia, W. Cui, H. Zhang, F. Xu, L. Sun, Y. Zou, H. Chu, E. Yan, Synthesis of three-dimensional graphene aerogel encapsulated n-octadecane for enhancing phase- change behavior and thermal conductivity, J. Mater. Chem. A 5 (2017) $15191-15199$.

[16] G.Q. Qi, C.L. Liang, R.Y. Bao, Z.Y. Liu, W. Yang, B.H. Xie, M.B. Yang, Polyethylene glycol based shape-stabilized phase change material for thermal 
energy storage with ultra-low content of graphene oxide, Sol. Energy Mater. Sol. Cells 123 (2014) 171-177.

[17] G. Fang, H. Li, Z. Chen, X. Liu, Preparation and properties of palmitic acid/SiO 2 composites with flame retardant as thermal energy storage materials, Sol. Energy Mater. Sol. Cells 95 (2011) 1875-1881.

[18] A. Sarı, A. Biçer, Thermal energy storage properties and thermal reliability of some fatty acid esters/building material composites as novel form-stable PCMs, Sol. Energy Mater. Sol. Cells 101 (2012) 114-122.

[19] X.H. Liang, Y.Q. Guo, L.Z. Gu, E.Y. Ding, Crystalline-amorphous phase transition of poly(ethylene glycol)/cellulose blend, Macromolecules 28 (1995) $6551-6555$.

[20] C. Chen, L. Wang, Y. Huang, Electrospun phase change fibers based on polyethylene glycol/cellulose acetate blends, Appl. Energy 88 (2011) 3133-3139.

[21] L. Wang, D. Meng, Fatty acid eutectic/polymethyl methacrylate composite as form-stable phase change material for thermal energy storage, Appl. Energy 87 (2010) 2660-2665.

[22] M. Mu, P.A.M. Basheer, W. Sha, Y. Bai, T. McNally, Shape stabilised phase change materials based on a high melt viscosity HDPE and paraffin waxes, Appl. Energy 162 (2016) 68-82.

[23] C. Chen, K. Liu, H. Wang, W. Liu, H. Zhang, Morphology and performances of electrospun polyethylene glycol/poly (dl-lactide) phase change ultrafine fibers for thermal energy storage, Sol. Energy Mater. Sol. Cells 117 (2013) 372-381.

[24] W. Hu, X. Yu, Encapsulation of bio-based PCM with coaxial electrospun ultrafine fibers, RSC Adv. 2 (2012) 5580-5584.

[25] Z. Chen, J. Wang, F. Yu, Z. Zhang, X. Gao, Preparation and properties of graphene oxide-modified poly(melamine-formaldehyde) microcapsules containing phase change material n-dodecanol for thermal energy storage, J. Mater. Chem. A 3 (2015) 11624-11630.

[26] C. Chen, W. Liu, Z. Wang, K. Peng, W. Pan, Q. Xie, Novel form stable phase change materials based on the composites of polyethylene glycol/polymeric 
solid-solid phase change material, Sol. Energy Mater. Sol. Cells 134 (2015) $80-88$.

[27] B. Tang, L. Wang, Y. Xu, J. Xiu, S. Zhang, Hexadecanol/phase change polyurethane composite as form-stable phase change material for thermal energy storage, Sol. Energy Mater. Sol. Cells 144 (2016) 1-6.

[28] Y. Zhang, L. Wang, B. Tang, R. Lu, S. Zhang, Form-stable phase change materials with high phase change enthalpy from the composite of paraffin and cross-linking phase change structure, Appl. Energy 184 (2016) 241-246.

[29] Z. Liu, B. Wu, X. Fu, P. Yan, Y. Yuan, C. Zhou, J. Lei, Two components based polyethylene glycol/thermosetting solid-solid phase change material composites as novel form stable phase change materials for flexible thermal energy storage application, Sol. Energy Mater. Sol. Cells 170 (2017) 197-204.

[30] C. Chen, W. Liu, H. Wang, K. Lin, Synthesis and performances of novel solid-solid phase change materials with hexahydroxy compounds for thermal energy storage, Appl. Energy 152 (2015) 198-206.

[31] K. Peng, C. Chen, W. Pan, W. Liu, Z. Wang, L. Zhu, Preparation and properties of $\beta$-cyclodextrin/4,4'-diphenylmethane diisocyanate/polyethylene glycol ( $\beta$-CD/MDI/PEG) crosslinking copolymers as polymeric solid-solid phase change materials, Sol. Energy Mater. Sol. Cells 145 (2016) 238-247.

[32] C. Chen, W. Liu, H. Wang, L. Zhu, Synthesis and characterization of novel solid-solid phase change materials with a polyurethaneurea copolymer structure for thermal energy storage, RSC Adv. 6 (2016) 102997-103005.

[33] G. Narayanan, R. Aguda, M. Hartman, C.C. Chung, R. Boy, B.S. Gupta, A.E. Tonelli, Fabrication and characterization of poly( $\varepsilon$-caprolactone)/ Hp- $\beta$-cyclodextrin pseudorotaxane nanofibers, Biomacromolecules, 17 (2016) 271-279.

[34] Z. Liu, X. Fu, L. Jiang, B. Wu, J. Wang, J. Lei, Solvent-free synthesis and properties of novel solid-solid phase change materials with biodegradable castor oil for thermal energy storage, Sol. Energy Mater. Sol. Cells, 147 (2016)177-184. 
[35] J. Su, P. Liu, A novel solid-solid phase change heat storage material with polyurethane block copolymer structure, Energy Convers. Manag. 47 (2006) $3185-3191$.

Figure Captions

Scheme 1 Schematic route for the shape-stabilized CPCMs.

Figure 1 FTIR spectra of (a) neat Hp- $\beta$-CD, PEG, MDI, PU copolymer and (b) the CPCM series.

Figure 2 SEM micrographs of (a1, a2) PEG and (b1, b2) PU copolymer. 
Figure 3 SEM macrographs of the shape-stabilized CPCMs: (a) CPCM-30; (b) CPCM-40; (c) CPCM-50 and (d) CPCM-60.

Figure 4 XRD diffractograms of (a) Hp- $\beta$-CD, PEG, PU copolymer and (b) the CPCM series.

Figure 5 POM images at room temperature: (a) PEG; (b) PU copolymer; (c) CPCM-30; (d) CPCM-40; (e) CPCM-50 and (f) CPCM-60.

Figure 6 In-situ POM images of PEG, PU copolymer and CPCM-40 during heating. Figure 7 In situ POM images of PEG, PU copolymer and CPCM-40 during crystallization process (timing started when the temperature of samples decreased to $\left.40{ }^{\circ} \mathrm{C}\right)$.

Figure 8 DSC thermograms of PEG, PU copolymer and CPCMs: (a) the heating process; (b) the cooling process.

Figure 9 The phase change enthalpies and temperatures of PEG, PU copolymer, CPCMs and EC/PEG PCMs for (a) melting process and (b) crystallization process. Figure 10 Schematic of phase transition process of CPCMs.

Figure 11 Enthalpy efficiency of the CPCMs during (a) melting and (b) crystallization.

Figure 12 (a) SEM image of CPCM-60 after 1000 thermal cycles and (b) FTIR spectra of CPCM-60 before and after the thermal cycles.

Figure 13 (a) DSC thermograms and (b) the corresponding thermal property data of CPCM-60 following varied thermal cycles.

Figure 14 (a) TGA and (b) DTG curves of PEG, Hp- $\beta$-CD, PU copolymer and the CPCMs. 